\title{
Effect of shared decision-making education on physicians' perceptions and practices of end-of-life care in Korea
}

\author{
Byung Chul Yư ${ }^{1}$, Miyeun Han², Gang-Jee Ko ${ }^{3}$, Jae Won Yang ${ }^{4}$, Soon Hyo Kwon ${ }^{5}$, Sungjin Chung ${ }^{6}$, Yu Ah Hong ${ }^{7}$, \\ Young Youl Hyun ${ }^{8}$, Jang-Hee Cho ${ }^{9}$, Kyung Don Yoo ${ }^{10}$, Eunjin Bae ${ }^{11}$, Woo Yeong Park ${ }^{12}$, In 0 Sun ${ }^{13}$, Dongryul Kim ${ }^{14}$, \\ Hyunsuk $\mathrm{Kim}^{15}$, Won Min Hwang ${ }^{16}$, Sang Heon Song ${ }^{17}$, Sung Joon Shin ${ }^{18}$ \\ For further information for the authors'affiliations, see Additional information.
}

Background: Evidence of the ethical appropriateness and clinical benefits of shared decision-making (SDM) are accumulating. This study aimed to not only identify physicians' perspectives on SDM, and practices related to end-of-life care in particular, but also to gauge the effect of SDM education on physicians in Korea.

Methods: A 14-item questionnaire survey using a modified Delphi process was delivered to nephrologists and internal medicine trainees at 17 university hospitals.

Results: A total of 309 physicians completed the survey. Although respondents reported that $69.9 \%$ of their practical decisions were made using SDM, 59.9\% reported that it is not being applied appropriately. Only $12.3 \%$ of respondents had received education on SDM as part of their training. The main obstacles to appropriate SDM were identified as lack of time (46.0\%), educational materials and tools (29.4\%), and education on SDM (24.3\%). Although only a few respondents had received training on SDM, the proportion of those who thought they were using SDM appropriately in actual practice was high; the proportion of those who chose lack of time and education as factors that hindered the proper application of SDM was low.

Conclusion: The majority of respondents believed that SDM was not being implemented properly in Korea, despite its use in actual practice. To improve the effectiveness of SDM in the Korean medical system, appropriate training programs and supplemental policies that guarantee sufficient application time are required.

Keywords: Clinical decision-making, End-of-life care, Life-sustaining treatment, Patient-centered care, Physician preference, Shared decision-making

\footnotetext{
Received: March 29, 2021; Revised: June 4, 2021; Accepted: August 25, 2021

Correspondence: Sung Joon Shin

Division of Nephrology, Department of Internal Medicine, Dongguk University Ilsan Hospital, 27 Dongguk-ro, Ilsandong-gu, Goyang 10326,

Republic of Korea. E-mail: shine@med.dongguk.ac.kr

ORCID: https://orcid.org/0000-0002-0777-9278
}

Byung Chul Yu and Miyeun Han equally contributed to this work as co-first authors.

Copyright (C) 2022 by The Korean Society of Nephrology

(a) This is an Open Access article distributed under the terms of the Creative Commons Attribution Non-Commercial and No Derivatives License (http:// creativecommons.org/licenses/by-nc-nd/4.0/) which permits unrestricted non-commercial use, distribution of the material without any modifications, and reproduction in any medium, provided the original works properly cited. 


\section{Introduction}

Shared decision-making (SDM) is a process by which patients and healthcare providers share the best available evidence and contribute to medical decisions under the mutual agreement [1]. Patients and physicians consider appropriate treatment options and make decisions together and share responsibility for the final decision $[2,3]$.

Since Veatch [4] introduced the concept of "sharing of decision-making" in 1972, many studies on the efficacy of SDM in clinical practice have been conducted. Medical SDM is associated with improvement of treatment compliance, patient satisfaction, and patient quality of life [5-7]. In addition, SDM improves patients' knowledge of treatment options and reduces conflict in the decision-making process related to uncertainty about their own values. Moreover, it encourages patients to play a more active role in the decision-making process and improve their awareness of associated risks [8-10]. Systematic reviews of SDM-related patient preferences and physicians' perceptions have revealed that interest in SDM is increasing, with SDM now the preferred decision-making method of most patients and physicians $[11,12]$. As evidence of ethical adequacy and clinical effectiveness accumulates, many countries are policing the application of the SDM approach $[13,14]$. Various strategies for the effective adoption and application of SDM have been studied. Among these strategies, SDM education for health-care professionals has been the most studied and, although its effectiveness has shown heterogeneous results, is relatively effective $[15,16]$.

Korea's Act on Decisions on Life-Sustaining Treatment for Patients in Hospice and Palliative Care or at the Endof-Life came into effect in February 2018. The law allows life-sustaining treatment (LST) to be withdrawn or withheld based on the decision of the patient or his or her legal representative. However, the current law does not mention a specific decision-making method; its purpose is to respect and guarantee the patient's right to make decisions about LST. A "good death," based on the patient's preferences, wishes, and needs, is the goal of the Act. It has been suggested that SDM is related strongly to "good death" in providing end-of-life care $[17,18]$. Because SDM can have an effect on the decision to withdraw or withhold LST, it has assumed importance in Korea.

SDM remains an unfamiliar concept in the Korean med- ical system. Although a few studies of the perspectives of nephrologists on decision-making about end-of-life care, including LST and palliative care, have been conducted $[19,20]$, no nationwide survey of the perspectives of nephrologists regarding SDM and the effect of SDM education on decision-making regarding end-of-life care has been conducted in Korea. The purpose of this study is to identify clinical attitudes and experiences of nephrologists and internal medicine trainees related to SDM in decision-making-including decisions related to LST-and to determine the current status of and barriers to SDM, focusing on the effect of SDM education.

\section{Methods}

\section{Study population}

We conducted a cross-sectional survey of internal medicine residents, fellows, and professors in nephrology at 17 university hospitals. All participants were members of the Korean Society of Geriatric Nephrology. The survey period ranged from January 1 to August 31, 2020. The study was conducted in accordance with the principles of the Declaration of Helsinki, and clinical data from patients were obtained after approval of the Institutional Review Board of Daejeon St. Mary's Hospital (No. DC19QEDI0085). As the residents and fellows were interacting with vulnerable participants who might have been concerned about the disadvantages of refusing to participate in the study, the current study was conducted with consent exemption. In addition, the following measures were performed to protect vulnerable participants: 1) the purpose and methods of the study were supplied to the survey participants at an open presentation; 2) the location of copies of the questionnaires was made known to the survey participants in advance; and 3) participants were given a one-week response time and advised of the location of the questionnaire collection box in advance.

\section{Questionnaire development process}

This study was designed as part of the project to identify the perspectives and attitudes of SDM among Korean physicians on end-of-life care. The other element of the study involved medical oncologists and residents of in- 
ternal medicine (NECA-NA-19-008). The questionnaire development process is described elsewhere [21]. Briefly, it followed a three-round modified Delphi process, which is a well-known method for identifying collective opinions of experts [22]. The Delphi process is characterized by anonymity among participants, iterative feedback of group opinion, aggregation of group response, and expert input. A panel of six experts discussed survey items in round 1 after reviewing the relevant legislation on LST and published articles on the perspectives of physicians regarding SDM in various medical decisions, including LST. Selecting the questionnaire items related to attitudes toward SDM centered on the decision to withhold or withdraw of LST. Round 1 involved a panel of six experts discussing survey items. In rounds 2 and 3, researchers participated in online and offline meetings to modify and select appropriate questionnaire items through discussion and agreement. A final questionnaire item was adopted if the content validity index was deemed appropriate after an in-depth review of its clarity, accuracy, understandability, and suitability for research purposes by experts in law, medical ethics, and palliative care medicine [23].

\section{Questionnaire configuration items}

In the introduction on the first page of the questionnaire, an explanation of the purpose and method of the current study and a statement guaranteeing the confidentiality of respondents were supplied. The following virtual-patient example was provided before respondents chose the "usual" decision-making method: "A 40-year-old man is diagnosed with an illness and is going to be treated. There are two treatment options; both have the same survival rate but different benefits and harms. What is the best decision-making method from your perspective?" The decision-making methods from which respondents could choose are presented as detailed explanations of nameless concepts: paternalistic, informative, interpretative, and SDM. The paternalistic approach was defined as determining the patients' clinical situation independent of their values and presenting them with evidence supporting the treatment decision. SDM was defined as discussing the patients' health-related values with them and deliberating together using evidence-based information to decide on their treatment plan. The informative approach was defined as using evidence-based information to help the patients understand their health conditions and all possible treatment options so they can choose a treatment plan based on their values. The interpretative approach was defined as helping the patients understand their personal values and suggesting evidence-based treatment options that fit those values [1]. The survey comprised two sections: participant demographics (eight items and a total of 10 questions) and attitude toward SDM and a decision to withhold or withdraw of LST (seven items and a total 16 of questions including four open questions).

\section{Statistical analyses}

Descriptive characteristics of the study population were reported as means \pm standard deviations and as frequency counts with percentages for categorical and binary variables. Comparisons of differences between groups were made using Mann-Whitney and Kruskal-Wallis tests for continuous variables and either chi-square tests or linear-by-linear association for categorical variables, as appropriate. The survey included some questions in which multiple options could be selected, and the number of answers to every item did not always sum to $100 \%$. All statistical tests were two-sided, and the results were presented with $95 \%$ confidence intervals. We considered the p-values less than 0.05 to indicate statistical significance. All analyses were performed using IBM SPSS version 25 for Windows (IBM Corp., Armonk, NY, USA) or Graphpad Prism5 (GraphPad, Inc., La Jolla, CA, USA).

\section{Results}

\section{Study population}

Of the 342 questionnaires distributed, 321 were completed and returned, for a response rate of $93.9 \%$. The authors reviewed missing data in detail and determined that each missing variable and the reason for absence were not related. The respondents with missing responses were excluded from the analysis, leaving 309 respondents in the final study group.

Of these respondents, 174 (56.3\%) were male, and 178 (57.6\%) were between 30 and 39 years of age. There were 226 residents $(73.1 \%)$, followed by 51 professors (16.5\%) 
and 32 fellows (10.4\%). Professors were grouped into nephrologists $(n=51)$ and compared with trainees (fellows and residents, $\mathrm{n}=258$ ). When respondents were asked how many patients (both outpatients and inpatients) they treated in the week previous to filling out the questionnaire, most (46.6\%) answered 20 to 49 . When asked how many decisions they made for their patients according to the Act on Decisions on Life-Sustaining Treatment for Patients in Hospice and Palliative Care or at the End-of-Life in the last week, $67.0 \%$ answered fewer than 2 (Table 1). Respondents were divided into educated $(\mathrm{n}=38)$ and non-educated (n $=271$ ) groups based on whether they had received SDM education as part of their training. Those who reported that they did not receive SDM education or were not sure were assigned to the non-educated group. No differences in sex, age, and position were evident between the two groups, but the proportion of respondents who reported having received SDM was higher among trainees than among nephrologists $(\mathrm{p}=0.047)$ (Table 1$)$.

\section{Patterns in the decision-making process among physi- cians}

After reading the virtual-patient example, the most "usual"

Table 1. Demographics of the questionnaire respondents

\begin{tabular}{|c|c|c|c|c|}
\hline Variable & Overall & Educated & Non-educated & $p$-value \\
\hline No. of respondents & 309 & 38 & 271 & \\
\hline Sex & & & & $0.12^{\mathrm{a}}$ \\
\hline Female & $135(43.7)$ & $12(31.6)$ & $123(45.4)$ & \\
\hline Age (yr) & & & & $0.11^{\mathrm{b}}$ \\
\hline$<30$ & $90(29.1)$ & $14(36.8)$ & $76(28.0)$ & \\
\hline $40-49$ & $26(8.4)$ & $1(2.6)$ & $25(9.2)$ & \\
\hline $50-59$ & $9(2.9)$ & $1(2.6)$ & $8(3.0)$ & \\
\hline $60-65$ & $6(1.9)$ & $0(0)$ & $6(2.2)$ & \\
\hline Position & & & & $0.41^{\mathrm{b}}$ \\
\hline Resident (1st/2nd grade) & $148(47.9)$ & 16 (42.1) & $132(48.7)$ & \\
\hline Position group & & & & $0.047^{b}$ \\
\hline Trainee & $258(83.5)$ & $36(94.7)$ & $222(81.9)$ & \\
\hline Nephrologist & $51(16.5)$ & $2(5.3)$ & 49 (18.1) & \\
\hline No. of patients treated by respondent (/wk) & & & & $0.54^{\mathrm{b}}$ \\
\hline$<20$ & $53(17.2)$ & $7(18.4)$ & $46(17.0)$ & \\
\hline $20-49$ & $144(46.6)$ & $20(52.6)$ & $124(45.8)$ & \\
\hline $50-79$ & $54(17.5)$ & $5(13.2)$ & 49 (18.1) & \\
\hline $80-99$ & $18(5.8)$ & $1(2.6)$ & $17(6.3)$ & \\
\hline$\geq 100$ & $40(12.9)$ & $5(13.2)$ & $35(12.9)$ & \\
\hline No. of patients who made decisionsc (/wk) & & & & $0.48^{b}$ \\
\hline
\end{tabular}

Data are expressed as number only or number (\%). Because of rounding, percentages might not sum to $100 \%$.

The p-values obtained from ${ }^{a}$ chi-square test or ${ }^{b}$ linear-by-linear association test. ${ }^{\mathrm{C}}$ According to the Act on Decisions on Life-Sustaining Treatment for Patients in Hospice and Palliative Care or at the End-of-Life. 
decision-making method chosen by respondents was the informative approach (56.3\%), followed by SDM (33.0\%); while the paternalistic approach $(3.2 \%)$ was the least-used method. The proportion of "usual" decision-making methods selected by respondents did not differ between the educated and non-educated groups $(\mathrm{p}=0.83$ ) (Fig. 1; Supplementary Table 1, available online).

Shared decision-making application in decisions to withhold or withdraw life-sustaining treatment in clinical practice

Regarding the questionnaire items on the actual use of SDM, $64.3 \%$ of respondents reported its application in their usual practice (Supplementary Table 2, available online). Regarding whether respondents received SDM education as part of their undergraduate or postgraduate curriculum, $12.3 \%$ answered that they did receive it and $57.0 \%$ reported that they did not. The percentage of respondents who reported that they did not receive training on SDM was particularly high among professors and those in their 50s (96.1\% and $90.7 \%$, respectively) (Supplementary Table 3, available online). Only $2.3 \%$ of respondents reported being aware of the specific SDM model; the others were not aware or not sure. Regarding the questionnaire items seeking to determine whether SDM is implemented appropriately in decisions to withdraw or withhold LST in actual clinical practice, $40.1 \%$ reported that SDM was implemented properly, $16.2 \%$ reported it was not appropriate, while the rest answered that they were not sure (Fig. 2). The proportion of respondents who used SDM for LST-related decisions tended to be higher $(84.2 \%$ vs. $69.7 \%, \mathrm{p}=0.05)$ in the educated group compared with the non-educated group. More educated respondents and trainees indicated that SDM is being used appropriately in actual clinical practice compared with members of the non-educated group and nephrologists $(63.2 \%$ vs. $36.9 \%, \mathrm{p}=0.002$ and $43.8 \%$ vs. $21.6 \%, \mathrm{p}=$ 0.003 , respectively) (Supplementary Table 3, 4; available online). Respondents chose lack of time as the most common reason why SDM has not applied appropriately in actual clinical practice (46.0\%). With regard to "patient aspects," unrealistic needs of the family, high dependence on the physician, and ambiguous timing in the decision-making process were reported frequently $(36.9 \%, 31.4 \%$, and $28.8 \%$, respectively). With regard to "physician aspects," respon- dents frequently cited the lack of educational materials and tools and lack of training on the SDM method $(29.4 \%$ and $24.3 \%$, respectively) (Fig. 2). With regard to the factors that hindered proper application of SDM, respondents in the educated and non-educated groups reported "insufficient time" (29.4\% vs. $48.7 \%, \mathrm{p}=0.03)$ and "not trained in SDM" ( $7.9 \%$ vs. $26.6 \%, p=0.02$ ). A lower proportion of educated respondents compared with the non-educated reported "lack of educational materials and tools" (15.8\% vs. $31.4 \%$, $\mathrm{p}=0.07$; Supplementary Table 3). A higher proportion of nephrologists than trainees reported that the factors hindering proper application of SDM were "ambiguity of the timing of the decision" ( $43.1 \%$ vs. $26.0 \%, \mathrm{p}=0.02$ ), "differences in patient preferences" ( $29.4 \%$ vs. $11.6 \%, \mathrm{p}=0.002)$, and "lack of educational materials and tools" (51.0\% vs. $25.2 \%, \mathrm{p}<0.001$; Supplementary Table 4).

\section{Discussion}

Previous studies on patient and caregiver satisfaction with SDM in various clinical situations have revealed that patients who experienced SDM were more likely to report a sense of well-being, greater satisfaction, and less regret compared with those without such experience [24,25]. One of the most critical topics in end-of-life care, including LST, in nephrology is the initiation, withholding, and withdrawal of dialysis; various studies have been conducted on this topic [26-29], including recent domestic research $[19,20]$. In dialysis treatment decision processes, sufficient information exchange and sharing of the decision-making process between the doctor and the patient are associated with less patient regret [27-29]. This is consistent with the current trajectory of the general approach to SDM and could be the reason for the need to establish appropriate SDM in the field of nephrology.

Respondents reported that the SDM approach was the most used decision-making method in actual clinical practice. However, most respondents reported that they did not receive adequate training on SDM and were not aware of a specific SDM model. Most also believed that SDM was not being applied appropriately in actual clinical practice, and lack of time, educational materials, and appropriate tools were the most cited reasons. The proportion of those who assumed they were using SDM appropriately in actual clinical practice was higher in the educated group compared 


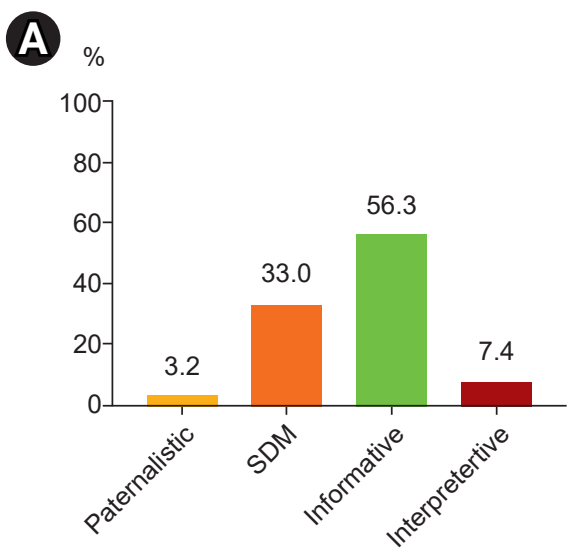

All respondents
B

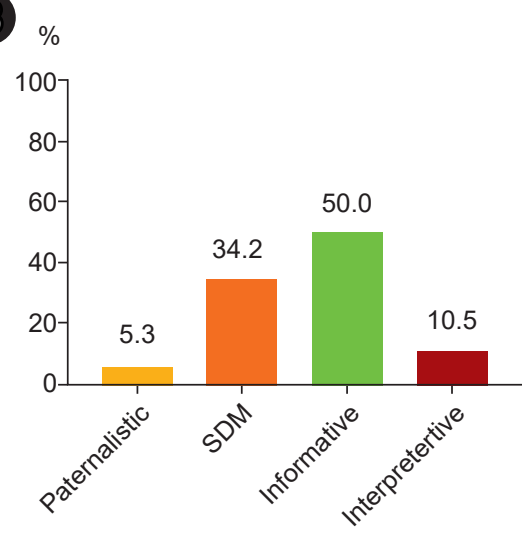

Educated group
(6)

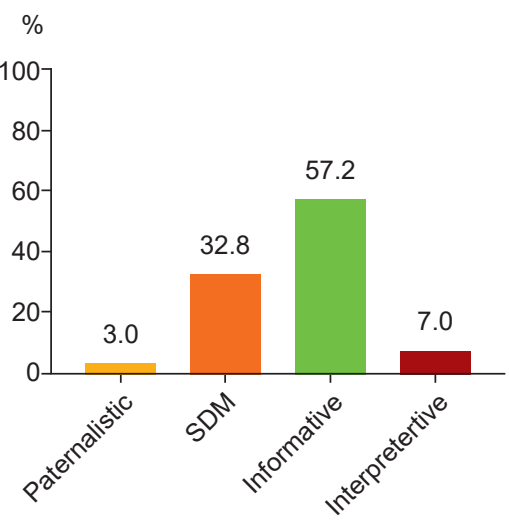

Non educated group

Figure 1. "Usual" decision-making approach of respondents after reading a fictional example of a patient in the decision-making process and detailed explanations of each decision-making approach. (A) All respondents. (B) Educated group. (C) Non-educated group.

SDM, shared decision-making.

Is SDM appropriately made in decision to withhold or withdraw of life-sustaining treatment in actual clinical practice?

$40.1 \%$

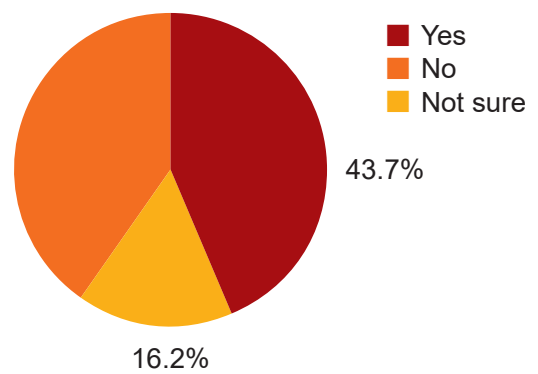

(c)

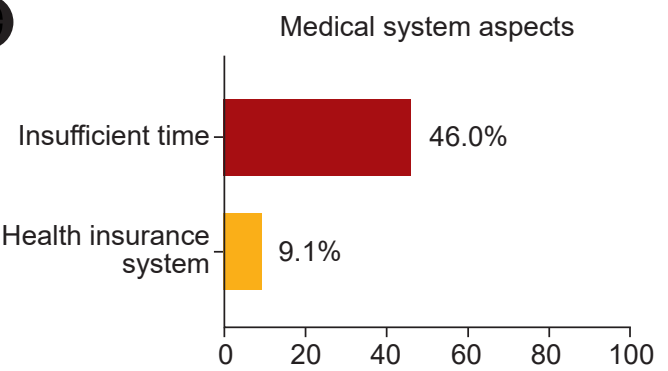

B

Physician aspects

Unrealistic needs of the family
Too dependent on the physician

The ambiguity of the timing of the decision

The lack of understanding of patients and caregivers

Differences in patient preference

Not being provided information about disease insight

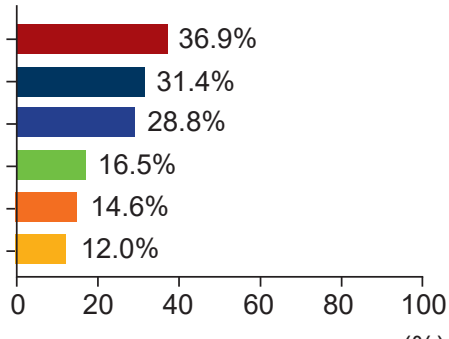

(\%)

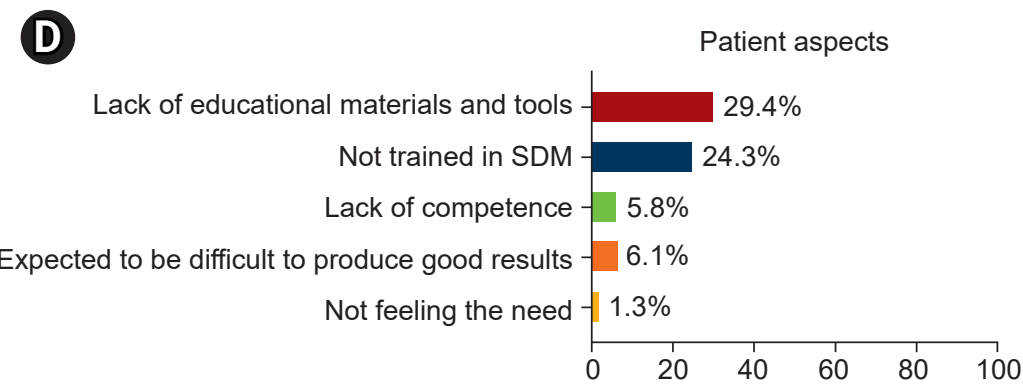

(\%)

Figure 2. Appropriateness of SDM in actual clinical practice and factors that hinder its proper application. (A) Response to the question "Is SDM appropriately made in decision to withhold or withdraw of life-sustaining treatment in actual clinical practice?" (B) Patient aspects. (C) Medical system aspects. (D) Physician aspects.

SDM, shared decision-making. 
with the non-educated group, while the proportion of those who chose lack of time and SDM education as factors hindering its proper application was lower.

Previous individual studies [30-32] and a meta-analysis [12] involving physicians have shown that, although their preference for SDM was consistently higher than other decision-making methods, the most preferred and most used methods differ in actual clinical practice. The current study results reflect those of previous studies in a larger context, but caution is needed when interpreting the results. In this study, the most used decision-making method in practice was the informative approach, including decisions to withhold or withdraw LST. This was based on respondents' selection of the method after they were presented with a detailed explanation of the nameless concept of each method. However, respondents reported that $64.2 \%$ of the decisions they make in actual practice involved SDM. The reasons for these conflicting responses-despite having been given detailed definitions of each decision-making method-could be insufficient understanding of the conceptual difference between methods. In other words, lack of education might account for these conflicting answers. Another possible explanation could be that many physicians assume that they are already using SDM at the decision-making stage, while in actual clinical practice they often do not know that their general decision-making method does not reflect SDM $[32,33]$.

When asked whether SDM is being applied properly in actual clinical practice, most physicians answered "no" or "unclear" and chose lack of time as the most common reason for not being able to apply it. Lack of time for physicians is a major obstacle to the SDM approach [34,35]. According to the Organisation for Economic Co-operation and Development (OECD) Health Statistics 2020, on the use of healthcare resources and utilization, number of annual outpatient visits per capita, average hospital stay per capita, and number of physicians per 1,000 population in Korea were the highest, second-highest, and third-lowest among OECD countries [36]. These data indicate that physicians in Korea have insufficient time to treat their patients compared with those in other OECD member countries. To overcome the lack of time, efforts to reduce time wasted due to improper SDM application by incorporating it into training curricula and providing sufficient education and continuous feedback from experts are needed. In this study, fewer respondents in the educated group chose "insufficient time" to explain the lack of proper application of SDM compared with the non-educated group. In the educated group, SDM was used more often in LST-related decisions, and the proportion of respondents who reported that SDM was used appropriately in actual clinical practice was higher compared with that in the non-educated group. A recent study also showed that, when a curriculum using standardized patients to teach the main concepts and techniques of SDM was applied to internal medicine residents, knowledge, attitude, and application ability of SDM were improved [37]. Considering that the respondents reported lack of education as the most common obstacle to proper application of SDM in "physicians' aspects," SDM education is essential to addressing the problems that hinder its proper application. A new scheduling algorithm that can allocate interview time for decision-making and an information system that actively supports physicians should be considered. Policy-makers should expend effort on developing policies that support allocating meaningful time for appropriate SDM [38].

Compared with trainees, nephrologists more frequently selected "ambiguity of the timing of the decision" and "differences in patient preferences" as factors that hinder proper SDM application. This can be attributed to two factors. First, nephrologists rather than trainees make the leading decisions as attending physicians. Second, based on their accumulated clinical experience, many nephrologists might believe that providing more objectified and diverse medical choices for patients in an appropriate doctor-patient relationship and allowing patients to make decisions that fit their values are superior options for achieving patient satisfaction and meeting physicians' legal responsibilities.

There are several limitations to the current study. As with similar questionnaire surveys, limitations related to nonresponse bias and representativeness of participants exist. The response rate was comparable to or higher than that of similar studies $[31,32]$. As this study was conducted as a multiregional and multicenter study in Korea, it provides an opportunity to understand the perspectives of Korean internal medicine residents and nephrologists. However, the results cannot be generalized to other internal medicine specialists because the specialist-level participants consisted only of nephrologists. Moreover, trainees accounted for $83.5 \%$ of the total study population, 
making it difficult to extrapolate their perception to that of all physicians. The results of this study showed a difference in the total number of patients treated by respondent per week between trainees and nephrologists. Trainees and nephrologists have different types and loads of tasks, making it unreasonable to generalize the results. To overcome this limitation, the authors divided the respondents into "trainees" and "nephrologists" and performed a subgroup analysis. Regarding the perception of SDM, the difference in perception between trainees and nephrologists was confirmed. However, because only $5.3 \%$ of the respondents had received SDM education, subgroup analysis according to position on importance and necessity of SDM education could not be performed. It is expected that further generalized results on the perception of and need for SDM education among physicians could be obtained if the results of the analysis were integrated with the aforementioned study results on medical oncologists and residents of internal medicine. Second, a discrepancy might exist between SDM perspectives and their actual application because physicians' perspectives on SDM were determined based solely on the questionnaire without verification of their actual application. In a recent study that recorded and analyzed clinical decisions made in actual clinics, SDM often was incomplete [39]. In addition, the content and level of SDM training can differ by medical school. Even if the students of these schools received the same training, a difference can be expected in understanding the educational content and the ability to implement SDM in clinical practice. One of the major limitations of this study is that it divided the participants into groups based on SDM education: those who received SDM education and those who did not, with the latter only having memories of receiving SDM education. However, despite the expectation that the content of training and the ability to apply SDM after training would vary among respondents, the group that reported having received training on SDM was more likely to report a higher rate of appropriate use of SDM in actual clinical practice. In addition, a lower proportion of respondents who chose lack of time and SDM education as a hindrance factor of SDM could be seen as disproving the importance of education for the proper settlement of SDM. To clarify this, we recommend an additional comparative study of groups who received or did not receive the same SDM education curriculum that includes verification of practical application ability. Third, there might be a desirability bias to meet social expectations because this study relied on physicians' self-reported knowledge. Physicians could have been reluctant to present opinions contrary to the social climate that encourages providing extensive medical information to patients and caregivers and engaging them in the decision-making process. Fourth, interpretation of the respondents' responses to questions about factors that hinder proper application of SDM was problematic because respondents were allowed to supply multiple answers. To overcome this, two independent researchers analyzed these data separately. Finally, the fidelity of each research subject's questionnaire was not evaluated by an objective method. Prior to administering the survey, we explained the purpose of the study in detail and asked the respondents to answer sincerely to produce accurate study results. We explained the survey questions in detail to prevent any missing values from respondents, and a sufficient question and answer session was conducted with the respondents who did not understand the survey questions. Considering that $3.7 \%$ missing data occurred despite these measures, we should have reviewed the fidelity of each research subject's questionnaire.

In conclusion, the majority of nephrologists and internal medicine trainees believed that SDM was not being implemented properly in Korea. Respondents cited a lack of time and education on SDM as the major obstacle to its proper application. Considering that dramatic changes to the medical system, including adjustments of health insurance fees, are needed to resolve this lack of time, appropriate training programs for SDM appear to be a realistic and feasible solution to properly strengthen SDM in the Korean medical system. The perceptions about SDM of nephrologists and internal medicine trainees and the findings of this study can be used to develop an appropriate SDM model in Korea.

\section{Additional information}

${ }^{1}$ Division of Nephrology, Department of Internal Medicine, Soonchunhyang University Bucheon Hospital, Bucheon, Republic of Korea

${ }^{2}$ Division of Nephrology, Department of Internal Medicine, Hallym University Hangang Sacred Heart Hospital, Hallym University College of Medicine, Seoul, Republic of Korea

'Division of Nephrology, Department of Internal Medicine, Korea University Guro Hospital, Korea University College of Medicine, 
Seoul, Republic of Korea

${ }^{4}$ Division of Nephrology, Department of Internal Medicine, Yonsei University Wonju College of Medicine, Wonju, Republic of Korea

${ }^{5}$ Division of Nephrology, Department of Internal Medicine, Soonchunhyang University Seoul Hospital, Seoul, Republic of Korea

${ }^{6}$ Division of Nephrology, Department of Internal Medicine, Yeouido St. Mary's Hospital, College of Medicine, The Catholic University of Korea, Seoul, Republic of Korea

${ }^{7}$ Division of Nephrology, Department of Internal Medicine, Daejeon St. Mary's Hospital, College of Medicine, The Catholic University of Korea, Daejeon, Republic of Korea

${ }^{8}$ Division of Nephrology, Department of Internal Medicine, Kangbuk Samsung Hospital, Sungkyunkwan University School of Medicine, Seoul, Republic of Korea

${ }^{9}$ Division of Nephrology, Department of Internal Medicine, Kyungpook National University Hospital, School of Medicine, Kyungpook National University, Daegu, Republic of Korea

${ }^{10}$ Division of Nephrology, Department of Internal Medicine, Ulsan University Hospital, University of Ulsan College of Medicine, Ulsan, Republic of Korea

${ }^{11}$ Division of Nephrology, Department of Internal Medicine, Gyeongsang National University Changwon Hospital, Changwon, Republic of Korea

${ }^{12}$ Division of Nephrology, Department of Internal Medicine, Keimyung University Dongsan Hospital, Keimyung University School of Medicine, Daegu, Republic of Korea

${ }^{13}$ Division of Nephrology, Department of Internal Medicine, Presbyterian Medical Center, Jeonju, Republic of Korea

${ }^{14}$ Division of Nephrology, Department of Internal Medicine, Incheon St. Mary's Hospital, College of Medicine, The Catholic University of Korea, Seoul, Republic of Korea

${ }^{15}$ Division of Nephrology, Department of Internal Medicine, Hallym University Chuncheon Sacred Heart Hospital, Hallym University College of Medicine, Chuncheon, Republic of Korea

${ }^{16}$ Division of Nephrology, Department of Internal Medicine, Konyang University Hospital, Daejeon, Republic of Korea

${ }^{17}$ Division of Nephrology, Department of Internal Medicine, Pusan National University Hospital, Pusan National University School of Medicine, Busan, Republic of Korea

${ }^{18}$ Division of Nephrology, Department of Internal Medicine, Dongguk University llsan Hospital, Dongguk University School of Medicine, Goyang, Republic of Korea

\section{Conflicts of interest}

All authors have no conflicts of interest to declare.

\section{Funding}

This work was supported in part by a Cooperative Research Grant 2019 from the Korean Society of Nephrology and the Soonchunhyang University Research Fund.

\section{Authors' contributions}

Conceptualization: BCY, MH, JWY, SHK, SC, YAH, YYH, DK, WMH, SJS

Data curation, Formal analysis, Investigation: All authors

Funding acquisition: YAH, BCY

Project administration: SJS

Writing-original draft: BCY, MH, SJS

Writing-review \& editing: GJK, SHS, JHC, KDY, EB, WYP, IOS, HK, BCY, MH, SJS

All authors read and approved the final manuscript.

\section{ORCID}

Byung Chul Yu, https://orcid.org/0000-0002-2686-1904 Miyeun Han, https://orcid.org/0000-0001-7304-2496 Gang-Jee Ko, https://orcid.org/0000-0001-8355-1083 Jae Won Yang, https://orcid.org/0000-0003-3689-5865 Soon Hyo Kwon, https://orcid.org/0000-0002-4114-4196 Sungjin Chung, https://orcid.org/0000-0002-9886-8339 Yu Ah Hong, https://orcid.org/0000-0001-7856-4955 Young Youl Hyun, https://orcid.org/0000-0002-4204-9908 Jang-Hee Cho, https://orcid.org/0000-0002-7031-5214 Kyung Don Yoo, https://orcid.org/0000-0001-6545-6517 Eunjin Bae, https://orcid.org/0000-0001-6890-4725 Woo Yeong Park, https://orcid.org/0000-0003-2662-2898 In O Sun, https://orcid.org/0000-0001-7245-3736 Dongryul Kim, https://orcid.org/0000-0002-1322-1887 Hyunsuk Kim, https://orcid.org/0000-0003-1889-253X Won Min Hwang, https://orcid.org/0000-0001-7548-6111 Sang Heon Song, https://orcid.org/0000-0002-8218-6974 Sung Joon Shin, https://orcid.org/0000-0002-0777-9278

\section{References}

1. Entwistle VA, Cribb A, Watt IS. Shared decision-making: enhancing the clinical relevance. J R Soc Med 2012;105:416-421.

2. Emanuel EJ, Emanuel LL. Four models of the physician-patient relationship. JAMA 1992;267:2221-2226.

3. Charles C, Gafni A, Whelan T. Shared decision-making in the medical encounter: what does it mean? (or it takes at least two to tango). Soc Sci Med 1997;44:681-692.

4. Veatch RM. Models for ethical medicine in a revolutionary age. What physician-patient roles foster the most ethical realtionship? Hastings Cent Rep 1972;2:5-7. 
5. Stewart MA. Effective physician-patient communication and health outcomes: a review. CMAJ 1995;152:1423-1433.

6. Joosten EA, DeFuentes-Merillas L, de Weert GH, Sensky T, van der Staak CP, de Jong CA. Systematic review of the effects of shared decision-making on patient satisfaction, treatment adherence and health status. Psychother Psychosom 2008;77:219226.

7. Rathert C, Wyrwich MD, Boren SA. Patient-centered care and outcomes: a systematic review of the literature. Med Care Res Rev 2013;70:351-379.

8. O'Connor AM, Bennett CL, Stacey D, et al. Decision aids for people facing health treatment or screening decisions. Cochrane Database Syst Rev 2009;(3):CD001431.

9. Stacey D, Bennett CL, Barry MJ, et al. Decision aids for people facing health treatment or screening decisions. Cochrane Database Syst Rev 2011;(10):CD001431.

10. Stacey D, Légaré F, Col NF, et al. Decision aids for people facing health treatment or screening decisions. Cochrane Database Syst Rev 2014;(1):CD001431.

11. Chewning B, Bylund CL, Shah B, Arora NK, Gueguen JA, Makoul G. Patient preferences for shared decisions: a systematic review. Patient Educ Couns 2012;86:9-18.

12. Pollard S, Bansback N, Bryan S. Physician attitudes toward shared decision making: a systematic review. Patient Educ Couns 2015;98:1046-1057.

13. Elwyn G, Laitner S, Coulter A, Walker E, Watson P, Thomson R. Implementing shared decision making in the NHS. BMJ 2010;341:c5146.

14. Delbanco T, Berwick DM, Boufford JI, et al. Healthcare in a land called PeoplePower: nothing about me without me. Health Expect 2001;4:144-150.

15. Légaré F, Stacey D, Turcotte S, et al. Interventions for improving the adoption of shared decision making by healthcare professionals. Cochrane Database Syst Rev 2014;(9):CD006732.

16. Légaré F, Adekpedjou R, Stacey D, et al. Interventions for increasing the use of shared decision making by healthcare professionals. Cochrane Database Syst Rev 2018;7:CD006732.

17. Siu HYH, Elston D, Arora N, et al. The impact of prior advance care planning documentation on end-of-life care provision in long-term care. Can Geriatr J 2020;23:172-183.

18. Witkamp FE, van Zuylen L, Borsboom G, van der Rijt CC, van der Heide A. Dying in the hospital: what happens and what matters, according to bereaved relatives. J Pain Symptom Manage 2015;49:203-213.

19. Hong YA, Chung S, Park WY, et al. Nephrologists' perspectives on decision making about life-sustaining treatment and palliative care at end of life: a questionnaire survey in Korea. J Palliat Med 2021;24:527-535.

20. Yun YS, Kwon SH, Jung JM, Jeon JS, Noh HJ, Han DC. Attitudes of dialysis unit physicians with regard to withholding and withdrawing dialysis. Korean J Nephrol 2009;28:13-18.

21. Kwon JH, Kim DY, Shin SJ, Park CY, Lee HJ. [Identifying the status of withwdrawal of life-sustaining treatment and exploration the Korean-style doctor-patient shared decision-making model]. Seoul: National Evidence-based Healthcare Collaborating Agency; 2020. In Korean.

22. Trevelyan EG, Robinson N. Delphi methodology in health research: how to do it? Eur J Integr Med 2015;7:423-428.

23. Yusoff MS. ABC of content validation and content validity index calculation. Educ Med J2019;11:49-54.

24. Shabason JE, Mao JJ, Frankel ES, Vapiwala N. Shared decision-making and patient control in radiation oncology: implications for patient satisfaction. Cancer 2014;120:1863-1870.

25. Flierler WJ, Nübling M, Kasper J, Heidegger T. Implementation of shared decision making in anaesthesia and its influence on patient satisfaction. Anaesthesia 2013;68:713-722.

26. Davison SN. End-of-life care preferences and needs: perceptions of patients with chronic kidney disease. Clin J Am Soc Nephrol 2010;5:195-204.

27. Gilman EA, Feely MA, Hildebrandt D, et al. Do patients receiving hemodialysis regret starting dialysis? A survey of affected patients. Clin Nephrol 2017;87:117-123.

28. Saeed F, Ladwig SA, Epstein RM, Monk RD, Duberstein PR. Dialysis regret: prevalence and correlates. Clin J Am Soc Nephrol 2020;15:957-963.

29. Tan E, Teo I, Finkelstein EA, Meng CC. Determinants of regret in elderly dialysis patients. Nephrology (Carlton) 2019;24:622-629.

30. Zeuner R, Frosch DL, Kuzemchak MD, Politi MC. Physicians' perceptions of shared decision-making behaviours: a qualitative study demonstrating the continued chasm between aspirations and clinical practice. Health Expect 2015;18:2465-2476.

31. Shepherd HL, Butow PN, Tattersall MH. Factors which motivate cancer doctors to involve their patients in reaching treatment decisions. Patient Educ Couns 2011;84:229-235.

32. Driever EM, Stiggelbout AM, Brand P. Shared decision making: physicians' preferred role, usual role and their perception of its key components. Patient Educ Couns 2020;103:77-82.

33. Joseph-Williams N, Lloyd A, Edwards A, et al. Implementing shared decision making in the NHS: lessons from the MAGIC programme. BMJ 2017;357:j1744. 
34. Joseph-Williams N, Elwyn G, Edwards A. Knowledge is not power for patients: a systematic review and thematic synthesis of patient-reported barriers and facilitators to shared decision making. Patient Educ Couns 2014;94:291-309.

35. Légaré F, Ratté S, Gravel K, Graham ID. Barriers and facilitators to implementing shared decision-making in clinical practice: update of a systematic review of health professionals' perceptions. Patient Educ Couns 2008;73:526-535.

36. Organisation for Economic Co-operation and Development (OECD). Health care resources: physicians [Internet]. Paris: OECD; 2020 [cited 2020 Dec 4]. Available from: https://stats.
oecd.org/Index.aspx?ThemeTreeid=9.

37. Rusiecki J, Schell J, Rothenberger S, Merriam S, McNeil M, Spagnoletti C. An innovative shared decision-making curriculum for internal medicine residents: findings from the University of Pittsburgh Medical Center. Acad Med 2018;93:937-942.

38. Pieterse AH, Stiggelbout AM, Montori VM. Shared decision making and the importance of time. JAMA 2019;322:25-26.

39. Braddock CH 3rd, Edwards KA, Hasenberg NM, Laidley TL, Levinson W. Informed decision making in outpatient practice: time to get back to basics. JAMA 1999;282:2313-2320. 